\title{
Angle calibration of emulsion read-out system for gamma-ray telescope by test beam
}

\author{
Yuya Nakamura* For GRAINE Collaboration ${ }^{\dagger}$ \\ Nagoya University \\ E-mail: ynakamuradflab.phys.naqova-u.ac.ip
}

\begin{abstract}
Nuclear emulsion is a three-dimensional tracking detector with a sub-micron spatial resolution. Recently, the applications of nuclear emulsion have been expanding into imaging fields because of the improvement in scanning speed. Emulsion read-out systems exhibit an optical systematic error in the angular measurement caused by the difference in refractive index. This error is conservatively estimated as $0.015 \mathrm{rad}$ at an absolute track angle of $45^{\circ}$, which is a non-negligible value for the gamma-ray telescope experiment. We have evaluated this error experimentally using a test proton beam of $400 \mathrm{GeV}$ at SPS/CERN. From the experimental results, the error decreased to $0.001 \mathrm{rad}$ at an absolute track angle of $45^{\circ}$, which we will report on in this paper.
\end{abstract}

The 3rd International Symposium on "Quest for the Origin of Particles and the Universe"

5-7 January 2017

Nagoya University, Japan

\footnotetext{
* Speaker.

${ }^{\dagger}$ Nagoya University, Kobe University, ISAS/JAXA, Okayama University of science, Aichi University of education, and Utsunomiya University
} 


\section{Introduction}

Nuclear emulsion is a three-dimensional tracking detector with a sub-micron spatial resolution. It has been used for the studies of elementary particle interactions and their topologies. Since the 1990s, automated emulsion read-out systems have been realized and the scanning speed has been improving. The latest system is the Hyper Track Selector (HTS), which scans at $4000 \mathrm{~cm}^{2} / \mathrm{h}$ [四]. Because of this, the applications of nuclear emulsion have been expanding into not only particle physics but also imaging fields (cosmic gamma-ray observation, muon-radiography, etc.).

The Gamma-ray Astro-Imager with Nuclear Emulsion (GRAINE) is a balloon-borne gammaray telescope project utilizing nuclear emulsion. The GRAINE aims to precisely observe gammaray sources in the $10 \mathrm{MeV}-100 \mathrm{GeV}$ energy region with a large aperture area $\left(10 \mathrm{~m}^{2}\right)$. Figure 1 shows the angular resolution of the emulsion telescope by a Monte Carlo (MC) simulation and some experimental data. A demonstration test was performed with a balloon-borne emulsion telescope in 2015, and the aim was to detect the brightest gamma-ray source, the Vela pulsar, in the 100$200 \mathrm{MeV}$ energy region[[]].

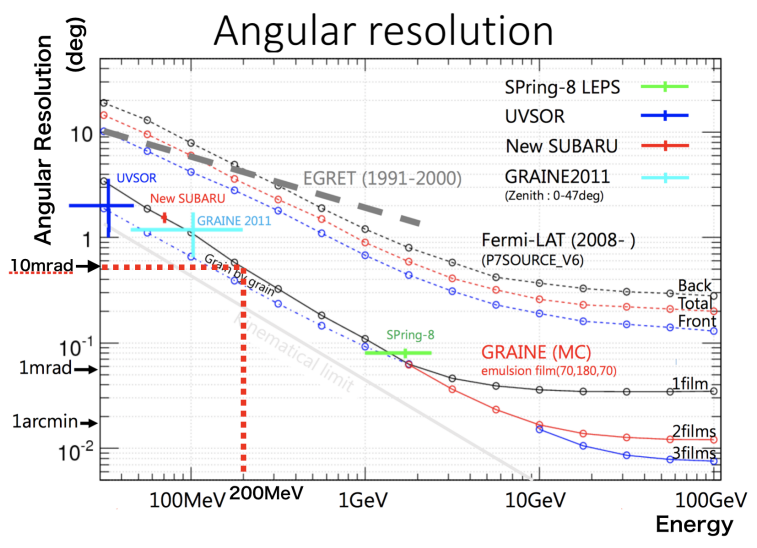

Figure 1: Angular resolution of gamma-ray telescopes. The solid lines represent the angular resolution of the emulsion telescope with MC and the points with error bars represent the experimental data. The dotted lines represent that of Fermi-LAT[B] and the broken lines represent that of EGRET[四]

Figure 2.a shows a cross-sectional view of the emulsion film. The emulsion films in the GRAINE 2015 experiment consisted of a 180- $\mu \mathrm{m}$-thick polystyrene film (base film) and $70-\mu \mathrm{m}$ thick emulsion layers applied to both sides. The HTS identifies tracks recorded in the emulsion layers. When tracks are detected in both layers, a virtual track in the base film, called a "base track", is defined by linking the passage positions at both surfaces of the base film. The base track is used for normal analysis because its position and angle are not significantly influenced by the shrinking or distortion of the emulsion.

The base track angle $\theta_{\text {scan }}$ exhibits a systematic error. The HTS measures the thickness of a base film thinner, as shown in Figure 2.b, because of the difference in refractive index between the emulsion and base film. As a result, $\theta_{\text {scan }}$ is larger than the absolute angle $\theta_{\text {abs }}$ (Figure 2.c). That relation is given by the following equations:

$$
\tan \theta_{\text {abs }} \simeq \frac{n_{\text {emul. }}}{n_{\text {base }}} \times \tan \theta_{\text {scan }}
$$




$$
k=\frac{n_{\text {emul. }}}{n_{\text {base }}}
$$

where $n_{\text {emul. }}$ and $n_{\text {base }}$ are the refractive indices of the emulsion and base film, respectively.

The value of $k$ is calculated as $\frac{1.51}{1.59}=0.95$. However, the refractive index depends on the wavelength and the objective lens of the HTS is more complex than that displayed in Figure 2. Thus, we conservatively estimate its error $\delta k$ as approximately 0.03 in the current analysis. Figure 3 shows the angular difference $\delta \theta\left(\arctan \left(\tan \theta_{\text {abs }}-k \times \tan \theta_{\text {scan }}\right)\right)$ caused by $\delta k$ as a function of $\theta_{\text {abs. }} \delta \theta$ exhibits a maximum value at $\theta_{\text {abs }} \simeq 45^{\circ}$. In the worst case $(\delta k=0.03), \delta \theta$ is $0.015 \mathrm{rad}$ at $\theta_{\mathrm{abs}}=45^{\circ}$. This value is a non-negligible systematic error for the GRAINE.

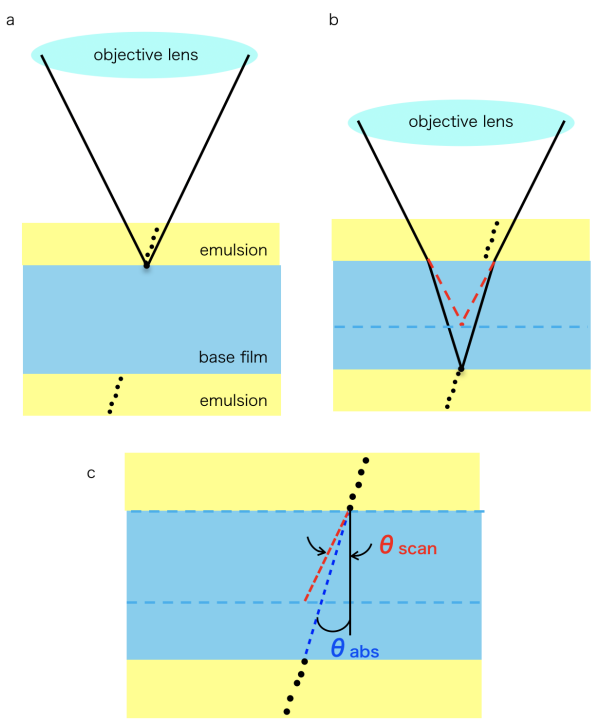

Figure 2: Cross-sectional view of emulsion film. Because of the difference in refractive index, $\theta_{\text {scan }}$ is systematically larger than $\theta_{\text {abs }}$.

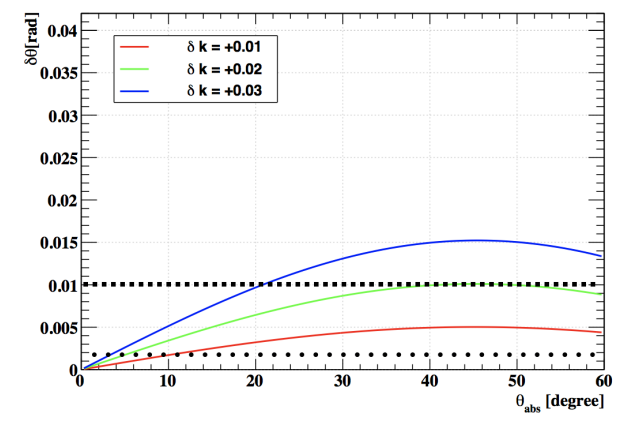

Figure 3: Calculated value of angular difference caused by $\delta k$. The black broken line represents the angular resolution of gamma-rays at $200 \mathrm{MeV}$ in the GRAINE. The dotted lines represent that at $1 \mathrm{GeV}$.

In this paper, we will report on the experimental evaluation of $k$ and $\delta k$ with a $400 \mathrm{GeV}$ proton beam at SPS/CERN.

\section{Angle Calibration by Beam Test at SPS/CERN}

\subsection{Setup and beam exposure}

Figure 4 shows an illustration of the experimental setup. The structure of the emulsion film is identical to that of the GRAINE, and three films $(5 \mathrm{~cm} \times 6.25 \mathrm{~cm})$ were vacuum-packed with a 5-mm-thick flat aluminum plate. We mounted this emulsion chamber onto a rotary table. The accuracy of the rotary table was measured as less than $1 \mathrm{mrad}$ by the FARO arm, which is a 3D coordinate measuring machine.

The beam test was conducted in Experimental Hall North1. We exposed the $400 \mathrm{GeV}$ proton beam to the emulsion chamber while varying the angle of incidence $\left(\theta_{\mathrm{abs}}\right)$. The beam intensity and beam profile were monitored by an upstream wire chamber and silicon detector telescope. The observed beam size had a length of $2 \mathrm{~cm}$ and width of $1 \mathrm{~cm}$. Table 1 summarizes the $\theta_{\text {abs }}$ and beam 


\begin{tabular}{|c|c|}
\hline $\begin{array}{c}\theta_{\mathrm{abs}}\left[^{\circ}\right] \\
\left(\tan \theta_{\mathrm{abs}}\right)\end{array}$ & $\begin{array}{c}\text { Beam density } \\
{\left[\times 10^{4} \text { tracks } / \mathrm{cm}^{2}\right]}\end{array}$ \\
\hline$-64(-2.0)$ & 1.28 \\
$-55(-1.4)$ & 1.34 \\
$-45(-1.0)$ & 1.27 \\
$-25(-0.47)$ & 1.25 \\
$0(0.0)$ & 1.25 \\
$25(0.47)$ & 1.24 \\
$45(1.0)$ & 1.32 \\
$55(1.4)$ & 1.27 \\
$64(2.0)$ & 1.27 \\
\hline
\end{tabular}

Table 1: Angle of incidence and beam density.

density. The last focus of the beam was located about $130 \mathrm{~m}$ upstream, so the angular size of the beam seen from a point of the detector was expected to be $\frac{0.5 \mathrm{~cm}}{130 \mathrm{~m}}=38 \mu \mathrm{rad}$.

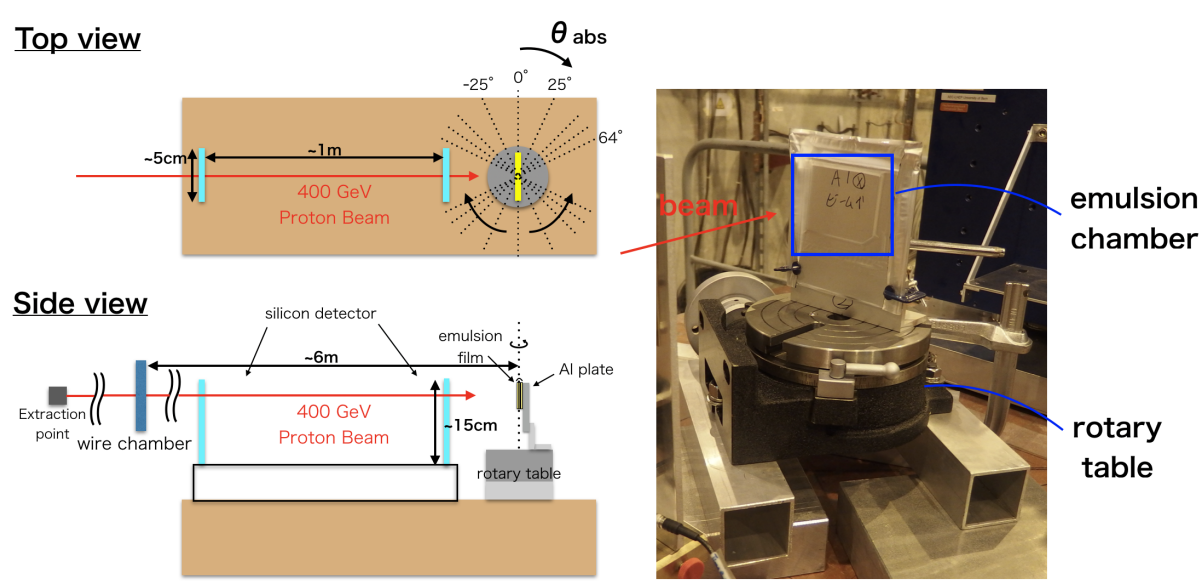

Figure 4: Experimental setup. The upper left image shows the top view, lower left image shows the side view, and right image shows the emulsion chamber mounted on the rotary table. We controlled the angle of incidence of the beam using the rotary table.

\subsection{Results and discussion}

The emulsion film was scanned by the HTS after development and the accepted angular range of scanning was $\left|\tan \theta_{X(Y)}\right|<1.8$. Figure 5 presents the scanned angle distributions. They display a very sharp peak at each angle we exposed. The mean value of each peak $<\tan \theta_{\text {scan }}>$ is defined as the average of the angles within the full-width at half-maximum.

Figure 6 displays the plot of $<\tan \theta_{\text {scan }}>$ vs. $\tan \theta_{\text {abs }}$ after correcting the rotation of the film while scanning. A line is fit by $Y=k \times X+p 1$. For this fit, the two angles at $\tan \theta_{\mathrm{abs}}=-1.4$ and 1.4 were not used, because some tracks near the edge of the accepted angular range of scanning were not recognized by the shrinking or distortion effect of the emulsion. The slope $k=0.9513 \pm 0.0019$ is consistent with the ratio of refractive index shown in section 1 . If $\delta k$ is 0.0019 , the calculated 
value of $\delta \theta$, as shown in Figure 3, is $0.00095 \mathrm{rad}$ at $\theta_{\mathrm{abs}}=45^{\circ}$, and it is sufficiently small for the GRAINE.
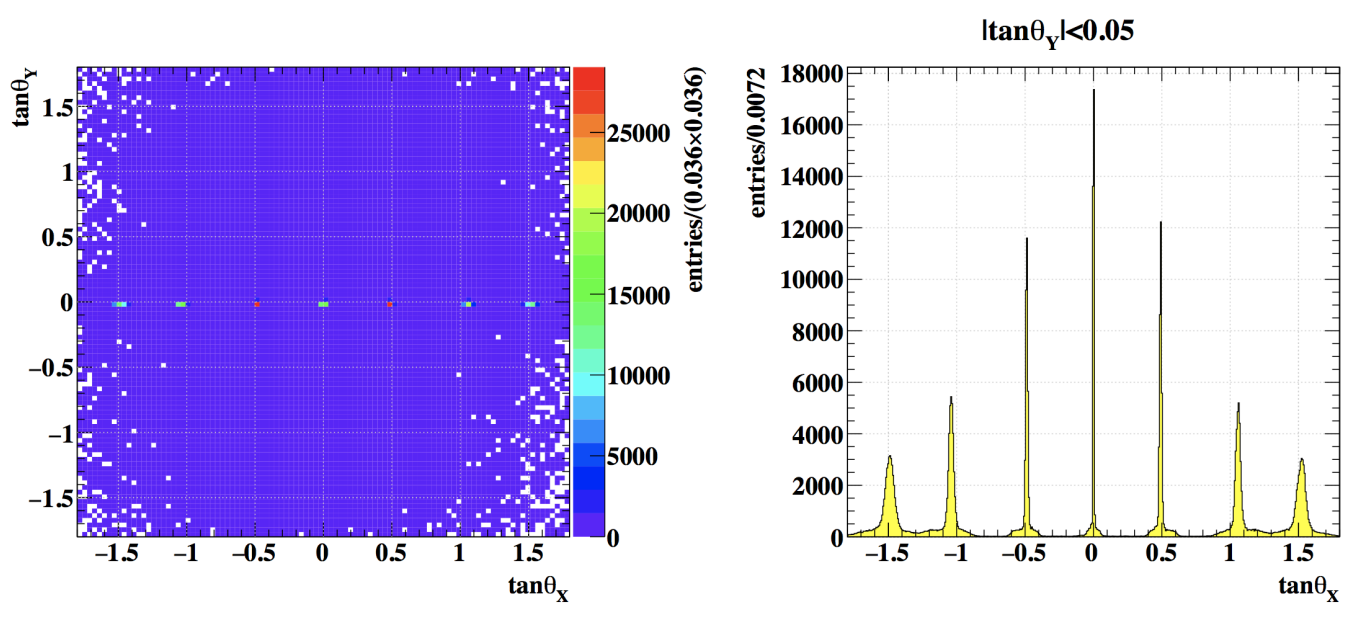

Figure 5: Scanned angle distributions.

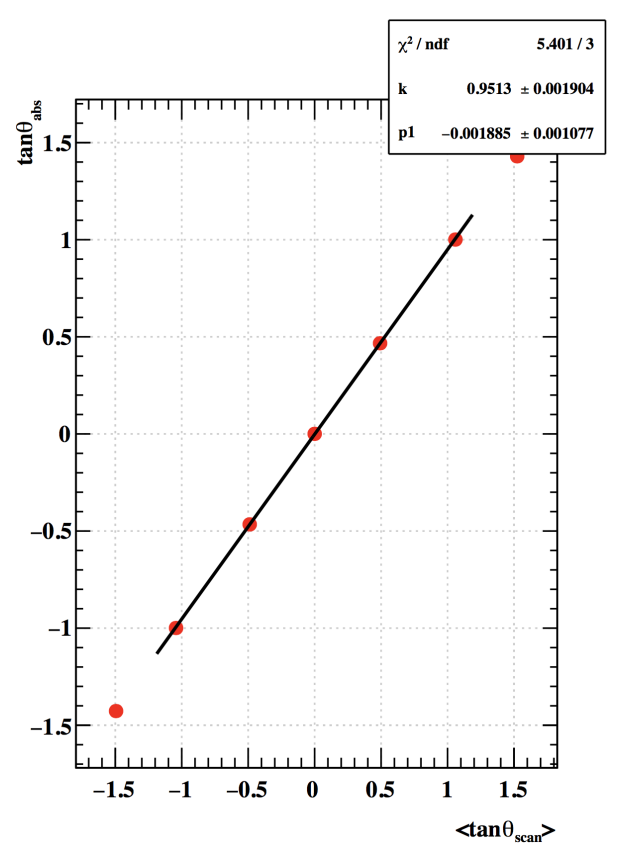

Figure 6: Comparison of absolute angle $\tan \theta_{\mathrm{abs}}$ and scanned angle $\tan \theta_{\text {scan }}$. The line indicates the result of the linear fit.

\section{Summary}

Recently, the applications of nuclear emulsion have been expanding into imaging fields such as GRAINE because of the improvement in scanning speed of automated emulsion read-out systems. 
Emulsion read-out systems exhibit an optical systematic error in the angular measurement caused by the difference in refractive index. This error was conservatively estimated as $0.015 \mathrm{rad}$ at $\theta_{\mathrm{abs}}=$ $45^{\circ}$. To understand the relation between $\theta_{\text {scan }}$ and $\theta_{\text {abs }}$, we evaluated this error experimentally. With the present experimental results, the error decreased to $0.001 \mathrm{rad}$ at $\theta_{\mathrm{abs}}=45^{\circ}$.

\section{References}

[1] M. Yoshimoto, et al., to be published in PTEP.

[2] S. Takahashi, et al., PTEP (2016) 073F01.

[3] https://www.slac.stanford.edu/exp/glast/groups/canda/archive/pass7v6/lat_Performance.htm

[4] D.J.Thompson, et al. Astrophysical Journal Supplement Series 86 (1993): 629-656. 